\title{
Effect of microwave irradiation on the electronic structure of $\mathrm{ZnO}$
}

\section{$\operatorname{AUTHOR}(\mathrm{S})$ :}

Yoshida, Kyohei; Sonobe, Taro; Zen, Heishun; Hachiya, Kan; Okumura, Kensuke; Mishima, Kenta; Inukai, Motoharu; ... Kii, Toshiteru; Masuda, Kai; Ohgaki, Hideaki

\section{CITATION:}

Yoshida, Kyohei ... [et al]. Effect of microwave irradiation on the electronic structure of ZnO. Journal of Physics and Chemistry of Solids 2015, 83: 47-51

\section{ISSUE DATE:}

2015-03-14

\section{URL:}

http://hdl.handle.net/2433/198552

\section{RIGHT:}

(c) 2015 Elsevier. Licensed under the Creative Commons Attribution-NonCommercial-No Derivatives 4.0 International http://creativecommons.org/licenses/by-nc-nd/4.0/. NOTICE: this is the author's version of a work that was accepted for publication in Journal of Physics and Chemistry of Solids. Changes resulting from the publishing process, such as peer review, editing, corrections, structural formatting, and other quality control mechanisms may not be reflected in this document. Changes may have been made to this work since it was submitted for publication. A definitive version was subsequently published in Journal of Physics and Chemistry of Solids, Volume 83, Pages 47-51.

doi:10.1016/j.jpcs.2015.03.013; 許諾条件により本文ファイルは2017-03-14に公開.; This is not the published version. Please cite only the published version.; この論文は出版社版でありません。引用の際には出版社版をご確認ご利用くだ さい。 
2 Kyohei Yoshida $^{1^{*}}$, Taro Sonobe ${ }^{2}$, Heishun. Zen ${ }^{1}$, Kan Hachiya ${ }^{3}$, Kensuke Okumura ${ }^{3}$,

3 Kenta Mishima ${ }^{3}$, Motoharu Inukai ${ }^{3}$, Hani $\mathrm{Negm}^{3}$, Konstantin Torgasin ${ }^{3}$, Mohamed

4 Omer $^{3}$, Ryota Kinjo ${ }^{4}$, Toshiteru Kii ${ }^{1}$, Kai Masuda ${ }^{1}$, and Hideaki Ohgaki ${ }^{1}$

$5{ }^{1}$ Institute of Advanced Energy, Kyoto University, Gokasyo, Uji, Kyoto 611-0011, Japan

62 Kyoto University Research Administration Office, Kyoto University,

7 Yoshida-honmachi, Sakyo-ku, Kyoto 606-8501, Japan

$8{ }^{3}$ Graduate School of Energy Science, Kyoto University, Yoshida-honmachi, Sakyo-ku,

9 Kyoto 606-8501 Japan

$10{ }^{4}$ RIKEN SPring-8 Center, 1-1-1, Kouto, Sayo-Cho, Sayo-Gun, Hyogo 679-5148, Japan

11 E-mail : k-yoshi@iae.kyoto-u.ac.jp Tel : +81-774-38-3423

Modifying the electronic structure may allow a bulk material to efficiently absorb radiation as well as excite and emit atomic plasma. The interaction between microwaves and metal oxides is investigated by analyzing the electronic structure of $\mathrm{ZnO}$ with and without microwave (MW) irradiation using absorption, photoluminescence (PL), and PL excitation (PLE) spectroscopies and utilizing an ultraviolet synchrotron light source. MW irradiation lowers the energy of the absorption edge of $\mathrm{ZnO}$ producing defects. Additionally, MW irradiation causes a resonantly enhanced change in the intensity and peak shift of the PL band. These phenomena indicate that the defects generated by MW irradiation change the electronic structure of $\mathrm{ZnO}$ and the electron transition process.

KEYWORDS: electronic structure; optical properties; semiconductors; inorganic compounds; oxides 
1

2

3

\section{Introduction}

Microwave (MW) heating is an advanced method for materials processing due to its unique advantages such as rapid and selective heating compared to conventional methods [1]. To date, several approaches have been implemented. For example, MW heating in the liquid phase has enhanced the catalytic activity of $\mathrm{ZnO}$ and $\mathrm{MgO}[2,3]$. It also can create $\mathrm{ZnO}$ architectures such as $\mathrm{ZnO}$ nanorods, wire-like, or flower-like structures [4,5]. Additionally, MW sintering has been reported to improve the electronic property of $\mathrm{ZnO}$ as a varistor for semiconductor application [6-8].

Sonobe et al. reported that the surface modification of $\mathrm{TiO}_{2}$ for enhancement of catalytic activity and the direct reduction without a reducing agent of $\mathrm{TiO}_{2}$ were available by MW treatment [9,10]. Similarly, the enhancement of the photocatalytic activity of $\mathrm{PbMoO}_{4}$ by MW irradiation has been reported[11]. Thus, MW technology has been used in advanced materials processing to improve the properties of functional materials.

MW heating of $\mathrm{ZnO}$ enhances the photocatalytic activity, synthesis of $\mathrm{ZnO}$ architectures, improvement of the capability as a varistor or thin film fabrication and so on $[2,6-8,12,13]$. In addition, the interaction between microwaves and $\mathrm{ZnO}$ has been investigated [14]. It was also confirmed that the MW irradiation did not induce the change of the crystallinity of $\mathrm{ZnO}$ [13]. In a previous study [14], a phenomenological model was constructed without exploring the detailed electronic structure in the bandgap which was created by MW irradiation. A phenomenological model is useful to design MW-induced $\mathrm{Zn} / \mathrm{O}$ atomic plasma excitations and emissions, or oxidation/reduction of bulk ZnO. The change in the physical properties such as the electronic structure by MW heating should be indispensable to elucidate this mechanism.

Moreover, the electronic structure is a significant parameter with respect to the capability of a functional material such as a photocatalyst or an electronic device. Therefore, the effect of MW irradiation on the electronic structure has been also investigated [1,5,15]. Previous research by our group [13] includes investigation of the changes in both the absorption and PL spectra with MW irradiation. The observed emergence of bandgap absorption and the change in the PL band suggest that MW-induced defects are created. However, the correspondence between the enhanced PL band and the bandgap absorption was not confirmed. Therefore, details of the electronic structures with MW irradiation such as the change in the electron transition process have yet to be investigated.

To apply MW heating technology for material processing, the objective of this research 
1 is to clarify the effect of MW irradiation on the electronic structural change of $\mathrm{ZnO}$.

2 This study employs absorption, photoluminescence (PL), and PL excitation (PLE)

3 spectroscopies to analyze the electronic structure of $\mathrm{ZnO}$ with $\mathrm{MW}$ irradiation. The

4 absorption spectra provide details about the energy shift of the absorption edge of $\mathrm{ZnO}$

5 through MW irradiation, while the PL spectra provide information not only about the

6 electronic structure of the material but also about the defects or impurities. In addition,

7 the PLE spectra help to understand the difference between the excitation mechanisms

8 with and without MW irradiation as well as provide insight on the electronic structure

9 and the electron transition process.

\section{Material and Methods}

12 Commercially available ZnO powder (1 g) (Wako Pure Chemical Industry, - $5 \mu \mathrm{m}$ 13 99.9\%) was pressed into a rectangular pellet $\left(27.0 \times 6.7 \times 1.5 \mathrm{~mm}^{3}\right)$ and sintered in air 14 by a furnace at $873 \mathrm{~K}$ for $2 \mathrm{~h}$ before MW irradiation. Then the sample exposed to 15 500-W MW irradiation for $10 \mathrm{~min}$ using reactor under vacuum conditions with a 16 vacuum pressure of about $1 \mathrm{~Pa}$ and a MW frequency of $2.45 \mathrm{GHz}$ (wavelength : 124 $17 \mathrm{~mm})$. Details about MW irradiation are reported elsewhere [13].

18 The absorption spectra were acquired with a UV-VIS-NIR spectrometer (JASCO, 19 V-670). BL7B beamline in UVSOR [16] was used as the light source for PL and PLE 20 spectroscopies. Figure 1 depicts the experimental setup for PL and PLE spectroscopies. 21 The photon energy of the excitation light was varied between $2.92 \mathrm{eV}$ (425 nm) and 223.54 eV (350 nm). A spectrometer (Acton Research Corporation, Spectra Pro-300i) and 23 a CCD cooled by liquid nitrogen (Princeton Instruments Inc, In/CCD-1340/100-EB/1) were used for PL detection. The exposure time, measurement temperature, and energy resolution of the excitation light were $10 \mathrm{~s}$, room temperature, and $0.02 \mathrm{eV}$ (FWHM) 26 respectively.

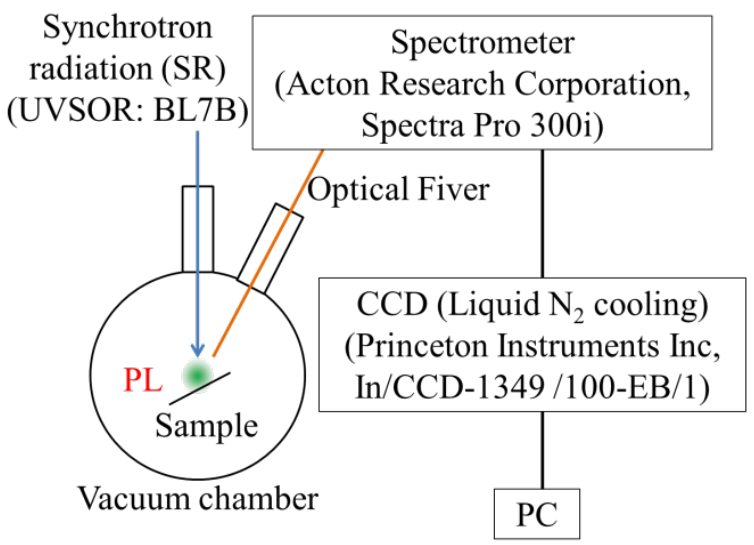

Fig. 1. Schematic of the setup for PL and PLE spectroscopies 
1 In addition, PL spectroscopy with a He-Cd laser (wavelength: $325 \mathrm{~nm}(3.81 \mathrm{eV})$, 2 power: $10 \mathrm{~mW}$, beam diameter: $1.0 \mathrm{~mm}$ : Kimmon IK5451R-E) was conducted at low 3 temperature (14 K) to investigate the band structure. The emission was observed by a 4 spectrometer (Zolix, Omni- $\lambda$ 300) with a CCD array (INTEVAC Mosir 350) and an 5 exposure time of $1 \mathrm{~s}$.

6 All data shown in this paper was corrected from single sample. To check the 7 sample-to-sample variation of the phenomenon, several samples were prepared and it

8 was confirmed that the same phenomenon was observed in samples prepared in same 9 condition.

\section{Results and Discussion}

$12 \quad 3.1$ Absorption spectra

13 Absorption spectroscopy was conducted with an emphasis on the absorption edge.

14 Figure 2 shows the absorption coefficient spectra. These spectra were converted from 15 the diffuse reflection spectra using Kubelka-Munk formula [9, 17], which is 16 expressed by Eq. (1) as

$$
\alpha / S=(1-R)^{2} / 2 R
$$

17 Here, $\alpha$ is the absorption coefficient, $S$ is the scattering coefficient, and $R$ is the diffuse

18 reflectance. $S$ is assumed to be constant, which is usually reasonable around the 19 absorption edge [9].

20 The energy of absorption edge of $\mathrm{ZnO}$ with or without MW irradiation were calculated 21 using Eq. (2) as [18, 19]

$$
(h v \alpha)^{2}=C\left(h v-E_{g}\right)
$$

22 Here, $h v$ is the photon energy ( $h$ is Planck's constant and $v$ is the frequency of light), $C$ 23 is a constant, and $E_{g}$ is the energy of the absorption edge. The blue arrows in Fig. 2 24 indicate the energy of the absorption edge determined from the absorption spectra. The 25 energies of the absorption edges of $\mathrm{ZnO}$ without and with $\mathrm{MW}$ irradiation are around $26 \quad 3.26 \mathrm{eV}$ and $3.22 \mathrm{eV}$, respectively. The absorption edge energy of the sample with MW 27 irradiation $(3.22 \mathrm{eV}$ ) is lower than that without MW irradiation (3.26 eV) (Phenomenon 28 1), suggesting that MW irradiation reduces the bandgap energy or creates new electronic 29 states below the conduction band. 


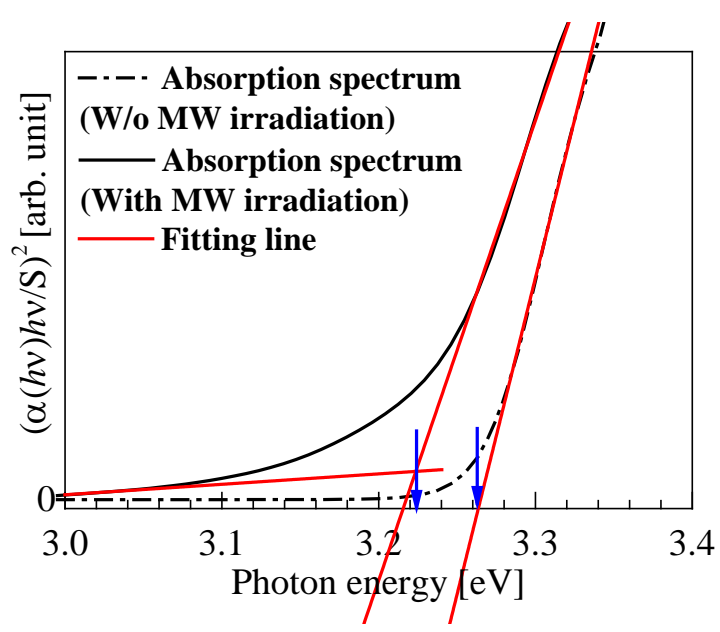

Fig. $2(h v \alpha / S)^{2}$ as a function of photon energy for the absorption edge of $\mathrm{ZnO}$ with and without MW irradiation. Red lines are visual guides for $h v \alpha \propto\left(h v-E^{\prime}\right)^{1 / 2}$ and to evaluate the absorption threshold ( $E^{\prime}=E_{g}$ for energy of the absorption edge)

\subsection{PL and PLE spectra at room temperature}

To confirm the defect-level energy, PL spectroscopies were investigated as functions of excitation energy. Figures 3 and 4 show the PL spectra for several excitation energies.

The broad peaks between $2.70 \mathrm{eV}$ and $3.50 \mathrm{eV}$ are the excitation light and the sharp peaks between $1.50 \mathrm{eV}$ and $1.80 \mathrm{eV}$ are inherently generated by the second order diffraction of the excitation light on the grating used to measure the PL spectra. The case without MW irradiation contains broad peak at $2.20 \mathrm{eV}$ and a band-edge emission at 3.20-3.30 eV (Fig. 4). To confirm that observed broad peaks are PL from the ZnO, a PL measurement with another material was conducted. The measurement was conducted on commercially available $\mathrm{TiO}_{2}$ powder (Furuuchi Chemical Corporation, TIC-72208B, -300M, purity:99.99\%) pressed into a rectangular pellet $(27.0 \times 6.7 \times 1.5$ $\mathrm{mm}^{3}$ ) of the same form with $\mathrm{ZnO}$ pellet and sintered in air by a furnace at $1273 \mathrm{~K}$ for 6 h. The crystal structure of the prepared $\mathrm{TiO}_{2}$ pellet was rutile [20]. The $\mathrm{TiO}_{2}$ pellet was irradiated the photon of $350 \mathrm{~nm}$ with the exposure time of $10 \mathrm{~s}$. The prepared $\mathrm{TiO}_{2}$ 0 sample is white color and barely shows the absorption in visible region [20]. As shown 1 in Fig. 3 (a), no peaks were observed in the case of $\mathrm{TiO}_{2}$ pellet. If the origin of the 2 observed broad peak is the measurement system such as the stray light of the excitation 3 light, the similar peak with higher intensity should be observed in the case of $\mathrm{TiO}_{2}$ 4 because the collected photon in spectrometer in the case of $\mathrm{TiO}_{2}$ is larger than that of the 5 case of $\mathrm{ZnO}$ as shown in Fig. 3 (b). These facts show that the observed broad peak is the 6 PL from $\mathrm{ZnO}$. As the result, the intensity of the broad peak at $2.20 \mathrm{eV}$ is nearly constant 27 regardless of the excitation energy (Phenomenon 2). Thus, the transition process of $\mathrm{ZnO}$ 
1 is independent of the excitation energy without MW irradiation.

2 However, in the case of the MW irradiation, the emission intensity of the broad peak

3 around $2.40 \mathrm{eV}$ strongly depends on the excitation energy, and the highest emission

4 intensity occurs with an excitation energy of $3.20 \mathrm{eV}$ (Fig. 4). This phenomenon implies

5 that the electron transition process was changed when the excitation energy was

6 changed.

7 PLE spectroscopy was conducted to understand the detailed electronic structure and

8 the electron transition process in the sample with MW irradiation. The upper panel and

9 right panel in Fig. 5 show the horizontal cross-section in which the excitation photons

10 emerge at $3.23 \mathrm{eV}$ and $3.35 \mathrm{eV}$, and the vertical cross-section whose photon energies in

11 the PL are $2.31 \mathrm{eV}$ and $2.20 \mathrm{eV}$ in the 2D graph, respectively. The small peak shift in

12 the central energy in the broad PL peak from $2.31 \mathrm{eV}$ (vertical red line) to $2.20 \mathrm{eV}$

13 (vertical black line) occurs when the excitation energy is changed from $3.23 \mathrm{eV}$ to 3.35

$14 \mathrm{eV}$. The broad peak (around $2.31 \mathrm{eV}$ ) has the highest PL when the excitation energy is

$153.23 \mathrm{eV}$. These results show that the emission intensity of the broad peak depends on the

16 excitation energy and that the maximum occurs at $3.23 \mathrm{eV}$ (Phenomenon 3). The broad

17 peak shifts from $2.31 \mathrm{eV}$ to $2.20 \mathrm{eV}$ as the excitation energy is elevated from $3.23 \mathrm{eV}$ to

$18 \quad 3.35 \mathrm{eV}$ (Phenomenon 4).

19 The peak at $2.20-2.40 \mathrm{eV}$ in $\mathrm{ZnO}$ is attributed to point defects such as oxygen

20 vacancies, zinc vacancies, zinc interstitials, oxygen interstitials, and oxygen-antisites

21 [21-27]. The electron transition process changes as the excitation energy is swept and

22 has a maximum of approximately $2.31 \mathrm{eV}$ when the excitation energy is $3.23 \mathrm{eV}$.

23 Therefore, the electron is trapped strongly at this energy level $(3.23 \mathrm{eV})$. Because the excitation energy resolution in the PLE is sufficiently high (around $0.02 \mathrm{eV}$ (FWHM)), the photon energy of the excitation light with a maximized PL intensity is coincident with the energy of the absorption edge $(3.22 \mathrm{eV})$ in Phenomenon 1. This fact supports the possibility that MW irradiation creates a new energy state at $3.22 \mathrm{eV}$.

If Phenomenon 1 of the absorption spectra and Phenomenon 3 of the PLE spectra originate from the energy shift of the conduction band, the electron transition process due to relaxation from the conduction band would not shift. However, excitation above $3.23 \mathrm{eV}$ of the sample with MW irradiation actually shifts the broad peak, indicating that the above assumption is incorrect. Hence Phenomena 1, 3, and 4 imply that MW irradiation creates a new energy state at $3.22 \mathrm{eV}$, which is below the conduction band. 

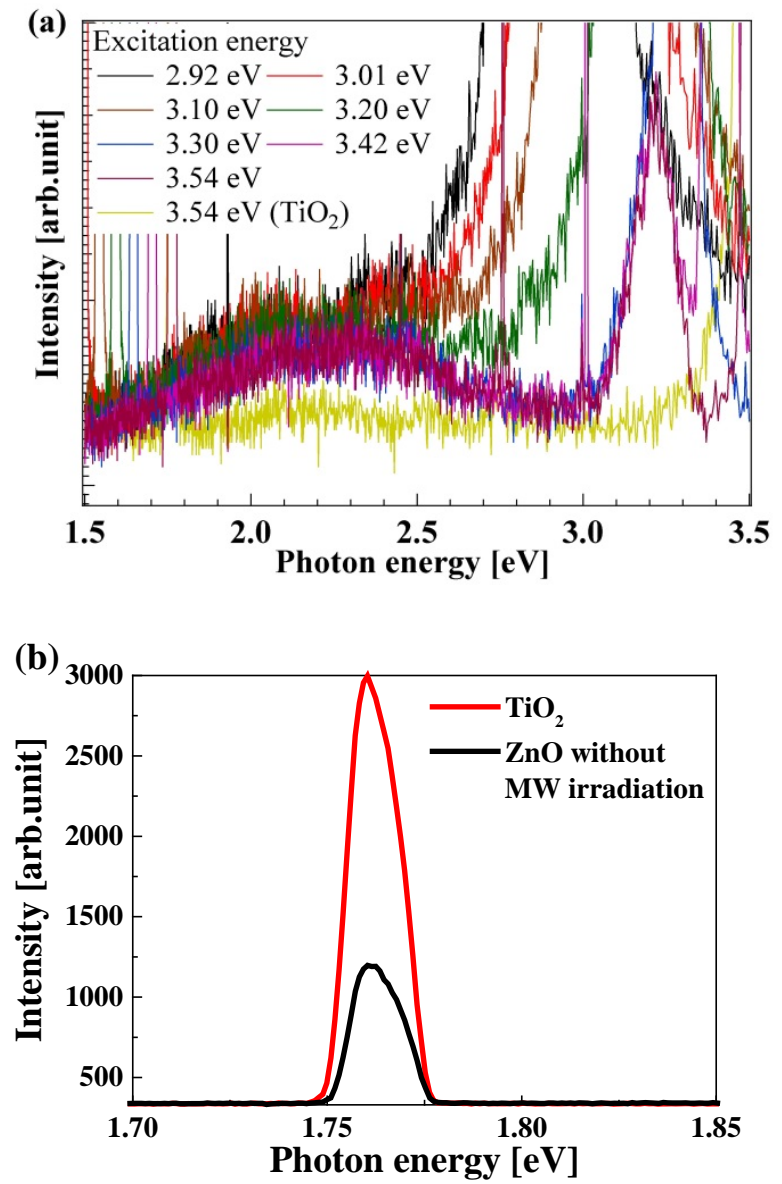

Fig. 3. (a) PL spectra of $\mathrm{ZnO}$ sintered at $873 \mathrm{~K}$ without $\mathrm{MW}$ irradiation $3.54 \mathrm{eV}(350 \mathrm{~nm})$ in the case of $\mathrm{TiO}_{2}$ and $\mathrm{ZnO}$ without MW irradiation.

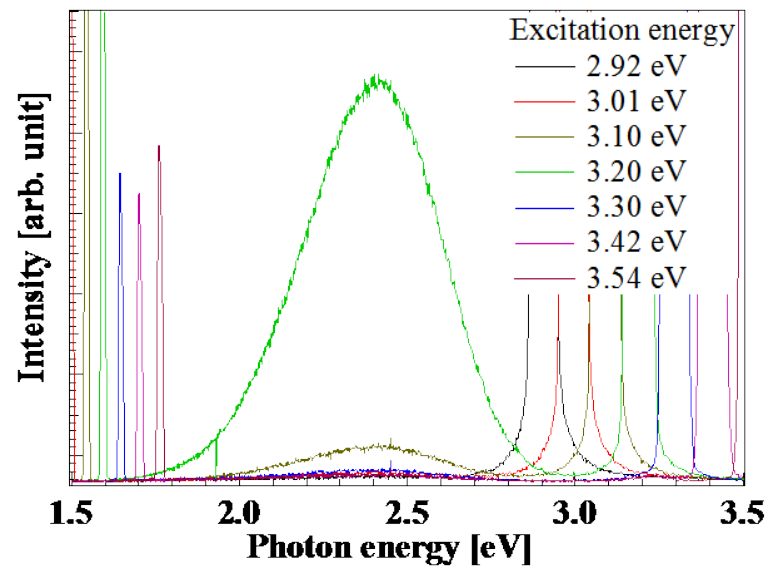

Fig. 4. PL spectra of $\mathrm{ZnO}$ sintered at $873 \mathrm{~K}$ with $\mathrm{MW}$ irradiation 


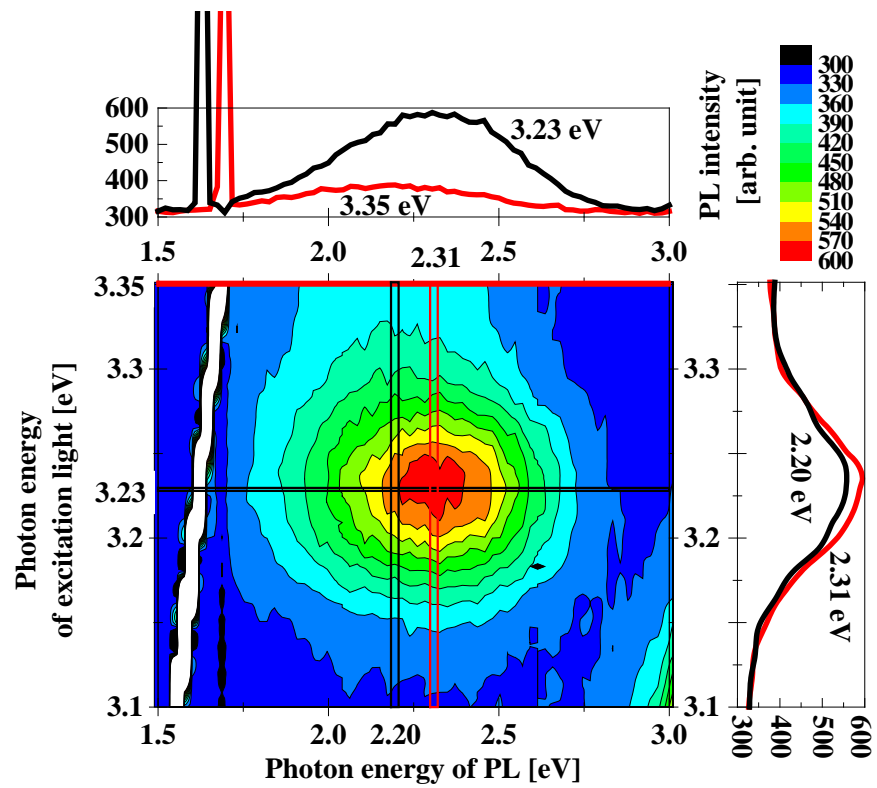

Fig. 5. PLE spectra of ZnO sintered at $873 \mathrm{~K}$ with $\mathrm{MW}$ irradiation

\subsection{PL spectra at low temperature}

PL measurements using a He-Cd laser at low temperature were conducted to understand the electronic structure near the bandgap energy. A band-edge emission with several peaks is observed in the specimen without MW irradiation (Fig. 6), and the center energies of those peaks were $3.36 \mathrm{eV}, 3.30 \mathrm{eV}, 3.23 \mathrm{eV}, 3.20 \mathrm{eV}, 3.16 \mathrm{eV}, 3.13 \mathrm{eV}$, and $3.09 \mathrm{eV}$ (Phenomenon 5). The peaks appear in intervals of $0.07 \mathrm{eV}(3.23 \mathrm{eV} \rightarrow 3.16$ $\mathrm{eV})$ and about $0.10 \mathrm{eV}(3.30 \mathrm{eV} \rightarrow 3.20 \mathrm{eV} \rightarrow 3.09 \mathrm{eV}$, or $3.23 \mathrm{eV} \rightarrow 3.13 \mathrm{eV})$. The peaks at $3.36 \mathrm{eV}, 3.30 \mathrm{eV}$ and $3.23 \mathrm{eV}$ in Fig. 7 are assigned to the exciton bound to an ionized donor, a two-electron transition of an exciton bound to a neutral donor, and a donor to an acceptor pair transition (DAP), respectively [28]. This spectrum is a typical phonon replica, and peaks appearing at $0.07 \mathrm{eV}$ intervals have been attributed to the phonon replicas of the longitudinal optical mode, while peaks appearing at $0.10 \mathrm{eV}$ intervals have been reported as the phonon replica induced by two phonons of the transverse optical phonon [28]. A phonon replica is observed in the PL spectrum of a material with a high purity and a low defect density because the PL spectrum of a material with low purity and high defect density is dominated by the emission from the defect level [29]. Therefore, these results confirm that the specimen without MW irradiation has a high purity and low defect density.

On the other hand, the band-edge emission of the $\mathrm{ZnO}$ with MW irradiation (Fig. 7) has two peaks with energies of $3.36 \mathrm{eV}$ and $3.32 \mathrm{eV}$ (Phenomenon 6), and a phonon replica is not observed in case of the PL spectrum with MW irradiation. The peak at $3.32 \mathrm{eV}$ is assigned as excitons bound to $\mathrm{Zn}$ vacancy [30]. Therefore, these observations 
1 demonstrate that the MW irradiation creates Zn-vacancy defects in $\mathrm{ZnO}$.

2 Both specimens with and without MW irradiation show an exciton emission at $3.36 \mathrm{eV}$

3 (Figs. 6 and 7). Because the exciton emission of each specimen is coincident with each

4 other [31], MW irradiation does not change the band structure outside of the bandgap.

5 Therefore, these results are also consistent with the creation of a new energy state

6 between the conduction and valance bands due to MW irradiation (Section 3.2).

7

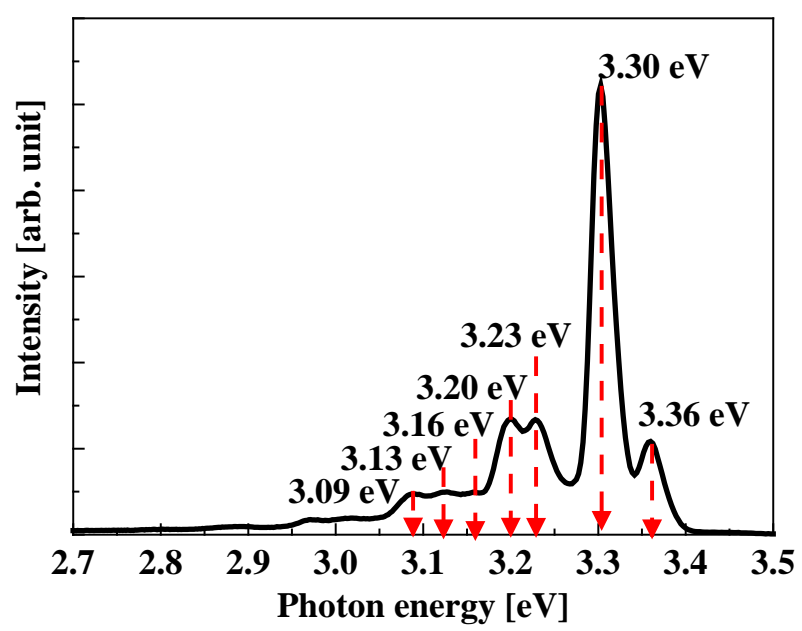

8

9 Fig. 6. PL spectra using a He-Cd laser (325 nm, $3.81 \mathrm{eV})$ at low temperature (14 K)

10 on the surface of $\mathrm{ZnO}$ without MW irradiation

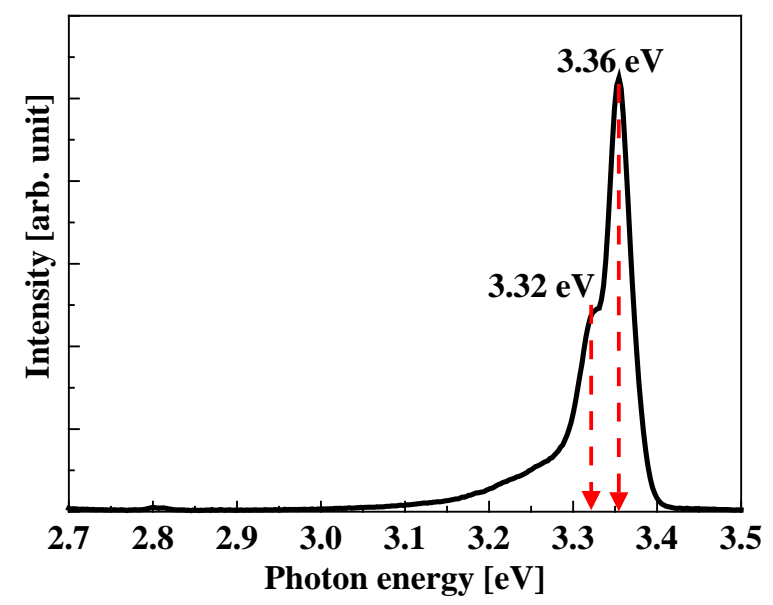

11

12 Fig. 7. PL spectra using a He-Cd laser (325 nm, $3.81 \mathrm{eV}$ ) at low temperature (14 K)

13 on the surface of $\mathrm{ZnO}$ with MW irradiation

14

15 3.4 Electronic structures of $\mathrm{ZnO}$ with and without $\mathrm{MW}$ irradiation

16 Figure 8 (a) and (b) show the energy diagram with and without MW irradiation at 
1 room temperature, respectively. The band at $2.20 \mathrm{eV}$ in Fig.3 reportedly originates from 2 the defect level [22, 24, 26]. In addition, the broad PL emission band, which is observed 3 for all excitation energies (2.92-3.42 eV) is similar to the case for a large Stokes-shift 4 PL. Therefore, the PL band at $2.20 \mathrm{eV}$ is a typical thermal creation of the deep level with a large lattice relaxation, and the electron is trapped around $3 \mathrm{eV}$. The fact that the PL intensity is independent of the excitation energy suggests that the trap level has a defined width (Fig. 8 (a)). Moreover, there is not an energy difference in the band-edge emissions between the two specimens at low temperature, suggesting that the bandgap energy at room temperature is $3.26 \mathrm{eV}$ for both specimens.

On the other hand, for $\mathrm{ZnO}$ with MW irradiation, an additional energy state is created near the conduction band due to the defect level generation by MW irradiation, which may be attributed to the plasma emission such as a zinc plasma and an oxygen plasma by MW irradiation or to the thermal effect induced by MW heating. Because, in the previous reports $[13,14]$, the plasma emission of zinc atom and oxygen atom was observed by microwave irradiation, and it shows that atoms of zinc and oxygen in $\mathrm{ZnO}$ are emitted from the sample. Therefore, this phenomenon shows the possibility that defects are created by the evaporation of the zinc atom and oxygen as plasma. In addition, the plasma emission shows that the sample was heated at high temperature over $2000{ }^{\circ} \mathrm{C}$ because the temperature around $2000^{\circ} \mathrm{C}$ is needed for decomposition, which is required to observe the plasma [13]. It is reported that defects are created when the sintering temperature is over $750{ }^{\circ} \mathrm{C}$ [32]. When the sample was partially heated over $2000^{\circ} \mathrm{C}$ by $\mathrm{MW}$ irradiation, temperature of the rest of the sample is also elevated to enough high temperature for defect creation. Therefore, as the second candidate of the cause of the defect creation, it is considered that defects are created by the thermal effect. However, additional investigations are required to clarify the detailed mechanism of the defect generation.

27 Because the band of the peak at $2.31 \mathrm{eV}$ in Fig.5 is also broad, the additional energy state by MW irradiation is also at a deep level with large lattice relaxation. In addition, the maximized intensity of the PL at a particular excitation energy $(3.23 \mathrm{eV})$ and the absorption edge at $3.22 \mathrm{eV}$ suggest that a clear trap level is generated around $3.22 \mathrm{eV}$.

In the case of the sample with MW irradiation, two route of the electron transition were considered (Fig.8 (b)). In Route 1 an electron transition occurs via the trap level.

33 In Route 2 an electron transition occurs via a trap level at $3.22 \mathrm{eV}$. Because the peak of the band shifts from $2.31 \mathrm{eV}$ to $2.20 \mathrm{eV}$, it is possible that the probability of the electron transition from the conduction band to the trap level at $3.22 \mathrm{eV}$ is lower than that from 
1 conduction band, Route 1 is favored to Route 2). These results indicate that MW 2 irradiation in $\mathrm{ZnO}$ creates a new energy state and modifies the electronic structure.

(a)

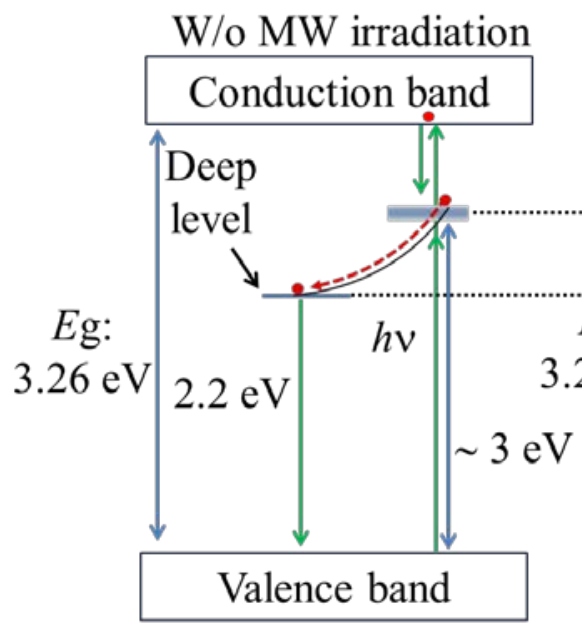

(b)

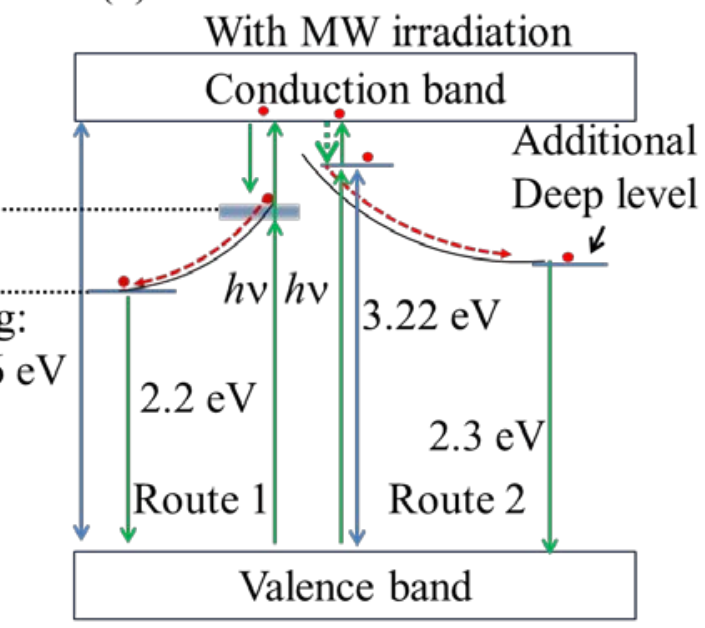

3

4 Fig.8 Schematic of the $\mathrm{ZnO}$ energy diagram at room temperature: (a) without and (b)

5 with MW irradiation

\section{Conclusion}

It was confirmed that MW irradiation affected the bandgap electronic structure of $\mathrm{ZnO}$, through conventional absorption spectroscopy, photoluminescence spectroscopy and photoluminescence excitation spectroscopy by an ultraviolet synchrotron light source. A new defect energy state slightly below $(\sim 0.04 \mathrm{eV})$ bandgap energy is created as a shallow energy level by MW irradiation to ZnO. The new MW irradiation-induced defects also alter the electron transition process. These findings indicate that this type of bandgap should allow a higher conductivity, which will enhance microwave-material 16 interactions. 


\section{$1 \quad 5$. Acknowledgment}

2 This work was partly supported by the "Energy Science in the Age of Global Warming" 3 of Global Center of Excellence (G-COE) program (J-051). The PL and PLE 4 measurements were supported by the Use-of-UVSOR Facility Program (BL7B 20125 2013) of the Institute for Molecular Science, Japan. We would like to thank all the staff 6 members of the UVSOR facility. Part of this study was supported by a Grant-in-Aid for 7 Scientific Research (C) (23561030) from the Japan Society for the Promotion of 8 Science. 


\section{Reference}

1 L.S.R. Rocha, R.C. Deus, C. R. Foschini, F. Moura, F. Gonzalez Garcia, A.Z. Simões, Mater. Res. Bull., 50, 12 (2014).

2 K. M. Parida, S. Parija, Solar Energy, 80, 1048 (2006).

3 H. Mirazaei, A. Davoodnia, Chin. J. Catal., 33, 1502 (2012).

4 M. K. Tsai, C. C.Huang, Y. C. Lee, C. S. Yang, H. C. Yu, J. W. Lee, S. Y. Hud, C. H. Chene, J. Lumin., 132, 226 (2012).

5 A. P. de Moura, R. C. Lima, M. L. Moreira, D. P. Volanti, J.W. M. Espinosa, M. O.

Orlandi, P. S. Pizani, J. A. Varela, E. Longo, Sol. St. Ion., 181, 775 (2010).

6 R. Subasri, M. Asha1, K. Hembram, G. V. N. Rao, T. N. Rao, Mater. Chem. Phys., 115, 677 (2009).

7 X. H. Zhu, Q. M. Hang, Micron, 44, 21 (2013).

8 S. Das, A. K. Mukhopadhyay, S. Datta, D. Basu, Bull. Mater. Sci., 31, 943 (2008).

9 T. Sonobe, J. Jitputti, K. Hachiya, T. Mitani, N. Shinohara, S. Yoshikawa, Jpn. J. Appl. Phys., 47, 8456 (2008).

10 T. Sonobe, T. Mitani, N. Shinohara, K. Hachiya, S. Yoshikawa, Jpn. J. Appl. Phys., 48, 116003 (2009).

11 D.B. Hernández-Uresti, A. Martínez-de la Cruz, J.A. Aguilar-GaribFacultad, Catalysis Today, 212, 70 (2013).

12 A. Kajbafvala, H. Ghorbani, A. Paravar, J. P. Samberg, E. Kajbafvala, S. K.

Sadrnezhaad, Superlattice Microst., 51, 512 (2012).

13 T. Sonobe, T. Mitani, K. Hachiya, N. Shinohara, H. Ohgaki, Jpn. J. Appl. Phys., 49, 080219 (2010).

14 T. Sonobe, K. Hachiya, T. Mitani, N. Shinohara, H. Ohgaki, J. Phys. Chem. Solids, 74, 837 (2013).

15 F. C. Romeiro, J. Z. Marinho, A. C. A. Silva, N. F. Cano §, N. O. Dantas, R. C. Lima, J. Phys. Chem. C, 117, 26222 (2013).

16 K. Fukui, H. Miura, H. Nakagawa, I. Shimoyama, K. Nakagawa, H. Okamura, T. Nanba, M. Hasumoto, T. Kinoshita, NIM A, 467-468, 601 (2001).

17 P. Kubelka, J. Opt. Soc. Am. 38, 448 (1948).

18 P. Yu and M. Cardona: Fundamentals of Semiconductors (Springer, Heidelberg, 1996).

19 K. Hachiya, T. Goto, R. Hagiwara, Electrochimica Acta, 53, 46 (2007).

20 Taro Sonobe, Tomohiko Mitani, Naoki Shinohara, Kan Hachiya, and Susumu

Yoshikawa, Jpn. J. Appl. Phys., 48, 116003 (2009)

21 S. A. M. Lima, F. A. Sigoli, M. Jafelicci Jr, M. R. Davolos, J. Inorg. Mater., 3, 749 (2001).

22 A. B. Djurišić, Y. H. Leung, K. H. Tam, L. Ding, W. K. Ge, H. Y. Chen, S. Gwo, Appl. Phys. Lett., 88, 103107 (2006).

23 D. Wang, H. W. Seo, C.-C. Tin, M. J. Bozack, J. R. Williams, M. Parka, N.

Sathitsuksanoh, An-jen Cheng, Y. H. Tzeng, J. Appl. Phys., 99, 113509 (2006).

24 M. Jiang, D. D. Wang, B. Zou, Z. Q. Chen, A. Kawasuso, T. Sekiguchi, Phys. Status

Solid A, 209, No. 11, 2126 (2012).

25 Q. X. Zhao, P. Klason, M. Willander, H. M. Zhong, W. Lu, J. H. Yang, Appl. Phys.

Lett., 87, 211912 (2005). 
26 T. M. Børseth, B. G. Svensson, A. Yu. Kuznetsov, P. Klason, Q. X. Zhao, M. Willander, Appl. Phys. Lett., 89. 262112 (2006).

27 B. Lin, Z. Fua, Y. Jia, Appl. Phys. Lett., 79, 943(2001).

28 S.A. Studenikina, M. Cociveraa, W. Kellnerb, H. Pascherb, J. Lumin., 91, 223 (2000).

29 S. Ramanathan, S. Bandyopadhyay, L. K. Hussey, and M. Muñoz, Appl. Phys. Lett., 89, 143121 (2006).

30 V. Sh. Yalishev, Y. S. Kim, X. L. Deng, B. H. Park, and Sh. U. Yuldashev, J. Appl. Phys., 112, 013528 (2012).

31 Ü. Özgür, Ya. I. Alivov, C. Liu, A. Teke, M. A. Reshchikov, S. Doğan, V. Avrutin, S.-J. Cho, and H. Morkoç, J. Appl. Phys., 98, 041301 (2005).

32 S.W. Xue, X.T. Zu, W.L. Zhou, H.X. Deng, X. Xiang, L. Zhang, H. Deng, Journal of Alloys and Compounds, 448, 21 (2008) 